\title{
The disruptive potential of drones
}

\author{
Stefanie Beninger ${ }^{1}$ (D) Karen Robson ${ }^{2}$
}

Accepted: 22 September 2020/Published online: 2 October 2020

(C) Springer Science+Business Media, LLC, part of Springer Nature 2020

\begin{abstract}
Drones are increasingly being used in marketing. Yet, despite rapidly growing adoption and incredible versatility, drones hardly feature in marketing research. This oversight is striking as their unique characteristics and the plethora of applications have major consequences for marketing. In particular, we outline how drones have implications for theory and practice in relation to business models, consumers, and society and public policy. We highlight these far-reaching consequences and provide a rich future research trajectory aimed to further theory development on the emerging phenomenon of drones.
\end{abstract}

Keywords Drones $\cdot$ Unmanned aerial vehicles $\cdot$ Marketing

\section{Introduction}

Drones - unmanned vehicles (e.g., airplanes, helicopters, submarines, or boats) that are controlled remotely by humans or artificial intelligence - are increasingly used around the world. Drones can be powered by battery, solar, or gas and vary in size - they can be small enough to fit in a hand or large enough to carry considerable amounts of cargo or people. These devices range in price from a few hundred to tens of thousands of dollars. The popularity of drones has grown dramatically in recent years, with millions of units sold globally and media headlines hailing the explosive demand for drones as a revolution.

Stefanie Beninger

stefanie.beninger@ie.edu

Karen Robson

krobson@uwindsor.ca

1 IE Business School, IE University, Calle de María de Molina, 11, 28006 Madrid, Spain

2 Odette School of Business, University of Windsor, 401 Sunset Ave, Windsor, Ontario N9B 3P4, Canada 
Drones have significant potential to impact the marketing function. Current uses range from small-scale applications in which drones carry advertising banners over public events, to potential wide-scale use to deliver essential services to vulnerable consumers, to the collection of massive amounts of information. Major brands such as Amazon, Google, Alibaba, 7-Eleven, Facebook, and the Red Cross have used or are testing drones in their marketing operations.

Despite increasing commercial and public attention on drones, there has been limited scholarly attention on them in business in general, and especially marketing. In one exception, Ferrell and Ferrell (2020) draw attention to integrating drone marketing into marketing education. In another, Khan et al. (2019) highlight some potential concerns with drone marketing in Pakistan. However, much more remains to be done. Drones are uniquely poised to disrupt traditional marketing practices due to their incredible versatility and ability to operate outside of conventional marketing approaches. Below, we discuss how drones change the nature of marketing and outline areas of marketing scholarship that would benefit from better understanding of the disruptive potential of drones.

\section{Drones and marketing}

Given how drone technology is developing and being adopted so rapidly, almost all areas of inquiry remain open. In particular, we suggest that research on drones should focus on three major areas: what are the implications of drones for (1) business models, (2) consumers, and (3) society and public policy? We elaborate on these general research areas below.

\subsection{Consequences for business models}

Drones change business models by providing alternatives to traditional processes or channel partners. For example, last-mile drone delivery is gaining attention, making the possibility of consumers encountering drones at their doorsteps - and therefore having drones become a customer-facing agent and touchpoint - a reality. This may be especially true for consumers who live in hard-to-reach areas, as drones need not travel using traditional infrastructure. Drones therefore make it possible for marketers to reach or communicate with customer segments that were previously unreachable. Drones are also being integrated into warehouse operations and retail settings. Walmart, for example, has filed a patent for using drones to move products between store departments. The use of drones in these ways impacts operational costs and provides benefits to customers. Thus, an important research avenue is investigating whether and how firms can (or already do) utilize drones to strengthen business models? What obstacles do marketers face in integrating drones into their operations?

One of the most direct ways that drones affect business models is through provisioning of products and services. Uber, for example, is working to develop peoplecarrying drones, and Chinese firm EHang's passenger drones are already in use. These new offerings could fundamentally shift mobility, including pricing, transportation structures, and norms. We suggest that worthwhile research trajectories involve investigating the following: how do drones impact existing products and services or the 
development of new ones? How do drone-based products and services impact business models?

As a distributed technology that can quickly and flexibly reconfigure and, increasingly, self-repair, it is likely that drones can bolster resilience, or the ability to maintain functions and structures in the face of changes (Allenby and Fink 2005). Research should explore the interplay between drones and resilience. Research that sheds light how drones impact the resilience of business models is especially needed given today's societal challenges; for example, drones have potential as a safe and resilient way to deliver products in the COVID-19 era.

\subsection{Consequences for consumers}

Drone marketing has wide-ranging implications for consumers, most notably in the areas of consumer adoption of drones, consumer privacy, and co-creation. Early surveys reveal consumer reservations about drones (Cohn et al. 2017), but little is known about how these reservations actually impact customer relationships or behavioral factors such as purchasing from companies that use drones in their operations. Research should investigate perceived risks (e.g., safety, security, privacy) in contrast to perceived benefits (e.g., efficiency, convenience, functional utility) of drone-enabled marketing, as well as the roles of social norms, familiarity, and knowledge of drones. In particular, understanding whether organizational drone use erodes consumer trust - a critical determinant of long-term customer relationships (Sirdeshmukh et al. 2002) would be informative. Key research questions include what are the critical determinants of consumer adoption of drones? How are adopters of drones similar or different to adopters of other technologies? How is adoption influenced by contextual factors?

One particularly important risk linked to drones is privacy. Concerns related to drone privacy infringement are already widespread (Ferrell and Ferrell 2020; Khan et al. 2018), with some concerned that drones can spy on them without their consent (see Chang et al. 2017). The emergence of smartphones changed notions of privacy in the early 2000s. Today, the audiovisual surveillance capabilities of drones are far more sophisticated than those of smartphones, and, unlike with smartphones, it is not possible to see "who" is behind a drone, which likely adds to the unease some already have with the technology. Drones can capture cellphone data and can, intentionally or incidentally, collect real-time photo or video information. Privacy concerns may be especially problematic in contexts besieged by corruption and weak privacy laws, and where unethically collected data could be used against already vulnerable consumers and communities. Furthermore, privacy research suggests that consumers are often willing to give up their personal information in order to receive benefits (see Martin and Murphy 2017). Yet, with drones, consumers may have (or simply perceive) less control over the information gathered and may also have more difficulty extracting benefits from it. Relevant research questions are how do drones impact the nature of privacy? How do drones differ from other technologies regarding privacy? Do consumers perceive the benefits of giving up their information to drone-based market research the same as they do for other forms of market research?

Consumer co-creation of value using drones is another promising research area. Drones operate bi-directionally and facilitate interactions between marketers and consumers. Some consumers may be motivated to improve marketing exchanges involving 
drones, and some companies are already leveraging co-creation opportunities, such as Airbus and their Cargo Drone Challenge, where consumers suggest commercial drone designs. Research questions include what role do consumers play as co-creators in drone-enabled marketing? What barriers to co-creation do consumers face?

\subsection{Consequences for society and public policy}

As drones impact business models and consumers, it follows that drones can have farreaching implications for social, environmental, and economic aspects of life, and thus for public policy. Drone-based marketing brings a complex array of societal benefits and harms. For example, there are risks of physical or environmental harm (e.g., due to crashes), with implications for public health. However, they can also improve access to products and services while bypassing environmentally and economically costly infrastructure (i.e., roads). The benefits of drones may be particularly significant in impoverished contexts (e.g., the base of pyramid), as these contexts are characterized by conditions of extreme scarcity and infrastructure shortcomings, leading to serious gaps in access to basic services such as healthcare or the internet. Drones offer a unique way to improve access to products and services in these contexts, thereby positively influencing society, as they can leapfrog over traditional and centralized offerings. For example, Facebook is developing a solar-powered drone that can provide internet to these unconnected areas, thus obviating the need for laying expensive cables. Research should explore the following: what societal, environmental, and economic benefits can be honed from using drones in marketing? What harms exist? What are the impacts of drones on impoverished contexts?

Public policy plays a role in mitigating risks and maximizing benefits through the adoption of best practices. Commercial drones currently operate under varying degrees of regulation, with opportunities for organizations and policymakers alike to make drones safer and more acceptable for consumers and communities. Research on the intersection of drones, marketing, and public policy is called for. Key questions include the following: what marketing policies maximize benefits to marketing companies, consumers, and society, while minimizing harms? How can regional, national, and international policies best coexist?

\section{Conclusion}

Drones are impacting consumers and companies in a meaningful way, and this impact will likely only grow in the coming years. Future work needs to do much more to uncover the wide-ranging theoretical implications of drone use in marketing as technology continues to mature. Certainly, there is ample opportunity to explore the implications of drones for business models, consumers, and society.

\section{References}

Allenby, B., \& Fink, J. (2005). Toward inherently secure and resilient societies. Science, 309(5737), 1034 1036. 
Chang, V., Chundury, P., \& Chetty, M. (2017, May). Spiders in the sky: user perceptions of drones, privacy, and security. In Proceedings of the 2017 CHI Conference on Human Factors in Computing Systems, 6765-6776.

Cohn, P., Green, A., Langstaff, M., \& Roller, M. (2017). Commercial Drones are Here: The Future of Unmanned Aerial Systems. McKinsey \& Company http://sonnycho.com/wp-content/uploads/2019/09/ McKinsey-Commercial-drones-are-here-The-future-of-UAS.pdf. Accessed 25 March 2020.

Ferrell, O. C., \& Ferrell, L. (2020). Technology challenges and opportunities facing marketing education. Marketing Education Review, 30(1), 3-14.

Khan, R., Tausif, S., \& Javed Malik, A. (2019). Consumer acceptance of delivery drones in urban areas. International Journal of Consumer Studies, 43(1), 87-101.

Martin, K. D., \& Murphy, P. E. (2017). The role of data privacy in marketing. Journal of the Academy of Marketing Science, 45(2), 135-155.

Sirdeshmukh, D., Singh, J., \& Sabol, B. (2002). Consumer trust, value, and loyalty in relational exchanges. Journal of Marketing, 66(1), 15-37.

Publisher's note Springer Nature remains neutral with regard to jurisdictional claims in published maps and institutional affiliations. 